\title{
e-Migrinter
}

17 | 2018

Enseigner les migrations internationales

\section{La diaspora Wenzhou en France et ses relations avec la Chine}

Thèse soutenue le 28 juin 2017 à l'Université de Poitiers

\section{Zhipeng Li}

\section{OpenEdition}

\section{Journals}

\section{Édition électronique}

URL : https://journals.openedition.org/e-migrinter/1165

DOI : 10.4000/e-migrinter. 1165

ISSN : 1961-9685

\section{Éditeur}

UMR 7301 - Migrinter

\section{Référence électronique}

Zhipeng Li, «La diaspora Wenzhou en France et ses relations avec la Chine », e-Migrinter [En ligne], 17 | 2018, mis en ligne le , consulté le 20 mai 2021. URL : http://journals.openedition.org/e-migrinter/1165 ; DOI : https://doi.org/10.4000/e-migrinter.1165

Ce document a été généré automatiquement le 20 mai 2021.

Tous droits réservés 


\section{La diaspora Wenzhou en France et ses relations avec la Chine}

Thèse soutenue le 28 juin 2017 à l'Université de Poitiers

Zhipeng Li

\section{RÉFÉRENCE}

Li, Zhipeng (2017) La diaspora Wenzhou en France et ses relations avec la Chine, Poitiers, Université de Poitiers

1 Au cours des dernières années, les migrations chinoises ont pris de l'ampleur donnant naissance à une diaspora de plus en plus importante. Cette thèse s'intéresse à la présence des migrants chinois originaires de la région de Wenzhou en France, qui s'est intensifiée à partir des années 1980 (Poisson, 2004 ; Auguin, 2009). Ce « sous-groupe » de la diaspora chinoise a notamment développé des activités économiques. L'objectif de cette thèse consiste à étudier l'organisation économique, sociale et spatiale des migrants de Wenzhou en France, principalement dans la région parisienne. Il s'agit également d'analyser les rapports économiques et sociaux que ceux-ci entretiennent avec la Chine et en particulier avec leur région d'origine.

2 En Chine, le «Modèle de Wenzhou » tel qu'il est défini par les chercheurs chinois correspond à une organisation économique particulière (Shi et Zhu, 2001). Ce modèle économique se caractérise par cinq éléments: 1) Des petites entreprises de type familial ; 2) Un financement par crédit de type «informel»; 3) Une tradition économique locale; 4) Des réseaux migratoires (internes et internationaux) pour la distribution de la production; 5) Une production visant l'échelon national et international. Ce modèle ainsi défini nous a servi de fil conducteur et de grille de lecture permettant la description de l'organisation économique Wenzhou en Chine, mais aussi en France. 
3 L'hypothèse centrale de la thèse est que le " Modèle de Wenzhou » en Chine, tel qu'il est identifié et analysé par les universitaires chinois, et le développement de l'entrepreneuriat chinois issu de Wenzhou en France sont étroitement liés.

Pour ce faire, nous interrogerons notamment l'idée d'une importation de ce modèle en France. S'il existe un « Modèle de Wenzhou » en Chine, retrouve-t-on ce même modèle en France? Notre recherche aborde les questions suivantes: D'une part, de quelles manières le «Modèle de Wenzhou ", issu du développement économique de la région de Wenzhou en Chine, transpose-t-il son influence sur les activités économiques de la diaspora Wenzhou en France? D'autre part, de quelles manières cette diaspora en France influence-t-elle le développement économique de la région de Wenzhou en Chine?

5 Pour répondre à ces questionnements, nous avons créé un dispositif méthodologique composé d'enquêtes permettant de démontrer les liens entretenus entre la diaspora Wenzhou et la Chine. Dans ce cadre, plusieurs études de terrain (2011-2015) ont été effectuées dans les lieux de départ et d'installation précédemment évoqués afin de saisir la multiplicité des points de vue des acteurs de cette diaspora.

6 L'essentiel des données présentées dans ce travail de recherche provient de ces enquêtes. Nous avons également traité les données statistiques des divers échelons du gouvernement local de Wenzhou, ainsi que d'autres sources disponibles sur place (documents officiels, revue de presse, etc.).

7 Dans le cadre des enquêtes de terrain, les entretiens ont été réalisés principalement avec des migrants chinois originaires de la région de Wenzhou. Les sources de nature démographique et économique sur cette population (de type censitaire) étant inexistantes en France puisque le recensement ne précise pas le lieu exact d'origine mais uniquement le pays, l'essentiel des données obtenues dans le cadre de cette recherche provient des enquêtes menées auprès des individus que nous avons sélectionnés. Bien que ces méthodes se soient souvent croisées dans la réalité, l'entretien semi-directif a été l'outil le plus employé dans notre travail. Une soixantaine d'entretiens (dont 33 auprès des entrepreneurs chinois d'origine de Wenzhou) ont ainsi été réalisés en Chine et en France auprès de chercheurs spécialisés dans l'étude de la diaspora chinoise, d'entrepreneurs, de travailleurs et d'universitaires originaires de Wenzhou, de représentants des gouvernements locaux chinois et français chargés des affaires étrangères et de dirigeants d'associations de Wenzhou.

8 L'analyse bibliographique montre que les migrations chinoises ont fait l'objet de nombreuses recherches dans le monde. Les travaux les plus nombreux concernent la présence chinoise dans les différents pays de l'Asie du Sud-Est au sens large (Freedman, 1957 ; Skinner, 1957 ; Wickberg, 1965 ; Purcell, 1965 ; G. Wang, 1991, 2006 ; Zhuang, 2009 ; Liu, 2009). Ils portent sur les questions identitaires liées à l'adaptation des migrants dans les différentes sociétés asiatiques, sur l'organisation économique ou encore sur l'histoire des migrations chinoises dans cette partie du monde. Les recherches sont également nombreuses en Amérique du Nord, l'étude des différents Chinatowns a été privilégiée notamment sur les questions d'adaptation des migrants chinois (Zhou, 1992). En Europe, les recherches sur la diaspora chinoise sont beaucoup moins nombreuses (Li, 2002; Thunø et Pieke, 2005 ; Polyzos, 2014). Les migrations chinoises en France ont fait l'objet de plusieurs recherches qui ont porté sur la dimension historique de leur implantation, sur les relations des immigrants chinois avec la société française, sur l'implantation géographique ou sur les activités 
économiques (Le Calloch, 1977 ; Poisson, 2004 ; Auguin, 2009 ; Pina-Guerassimoff, 2012 ; S. Wang, 2014 ; Ma Mung, 2014 ; Chuang, 2015). Ces recherches s'avèrent sommes toutes peu nombreuses, la diaspora chinoise en France reste alors peu étudié ${ }^{\text {. }}$

9 Cette thèse vise donc en premier lieu à combler ce déficit de connaissances. Elle s'inscrit également dans l'étude de recherche du laboratoire MIGRINTER qui prend en considération non seulement le pays d'installation des migrants, mais également le pays d'origine. En effet, les migrants sont considérés non pas dans un seul segment de l'espace migratoire comme, par exemple, celui dans lequel ils sont établis - ou celui d'où ils viennent - mais toujours replacé dans l'ensemble de l'espace migratoire qu'ils construisent : pays d'installation, bien sûr, mais aussi pays d'origine. C'est pour cela que notre recherche porte non seulement sur la diaspora Wenzhou en France mais aussi sur ses relations avec la Chine.

10 Par ailleurs, nous rappelons que la plupart des études sur les Chinois d'outre-mer (huaqiao huaren) réalisés en Chine privilégient les analyses s'appuyant sur les théories des migrations internationales de type push-pull ${ }^{2}$. Très peu de travaux ont entamé une théorisation de la "diaspora" (liusan zuqun) pour comprendre et analyser les migrations internationales chinoises comme cela a été fait dans le champ d'études francophones et anglophones menées sur la diaspora chinoise (Trolliet, 1994; Ma Mung, 2000 ; G. Wang, 2000 ; Kuhn, 2006). Nous avons souhaité envisager les migrants de Wenzhou comme une " diaspora » à part entière. À partir de ce point, cette thèse a permis de proposer une nouvelle approche pour qualifier le phénomène migratoire des Chinois.

11 La thèse s'organise en trois parties. La première partie repense la notion de " diaspora ", les théories des migrations internationales et les autres notions mobilisées par les travaux sur les migrations internationales chinoises qui permettent d'appréhender l'organisation économique de la diaspora Wenzhou. Nous décrivons la manière dont ces concepts et théories ont permis de formuler notre problématique (chapitre 1). Le chapitre suivant développe les méthodes d'enquête utilisées dans cette recherche pour saisir les relations entre les deux pôles migratoires des Wenzhou (chapitre 2). Les approches qui ont inspiré la conception de notre système d'investigation sont présentées dans ce chapitre, lequel précisera également la manière dont l'étude de terrain s'est déroulée, dans la région de Wenzhou et en France et les modes de collecte des données.

12 La deuxième partie est consacrée à l'analyse du « Modèle de Wenzhou » en Chine et de l'organisation économique de la diaspora chinoise en France. Dans cet objectif, une analyse du "Modèle de Wenzhou » et du développement économique de la région est proposée à partir des travaux de recherche existant en Chine, afin de saisir la spécificité du "Modèle de Wenzhou » (chapitre 3). Cette étude permettra quant à elle de démontrer à la fois comment s'est développée l'organisation entrepreneuriale dans la région parisienne, mais aussi comment celle-ci a évolué au point que le commerce de gros d'importation est devenu l'activité dominante (chapitre 4). Après avoir mis en lumière l'influence de ce modèle dans l'organisation économique de cette diaspora, nous mettons en évidence les éléments fondamentaux de ce modèle et les formes d'organisation de cette diaspora en France (chapitre 5).

13 La troisième partie est consacrée à une étude des interactions entre l'économie diasporique et la politique diasporique de l'État chinois. L'accent est d'abord mis sur la diaspora comme facteur de développement économique de la région de Wenzhou, en 
soulignant l'importance du crédit informel et de la distribution des produits grâce aux réseaux d'entrepreneurs, lesquels constituent les ressources économiques de la diaspora Wenzhou (chapitre 6). Les effets de la politique diasporique sont étudiés au travers des institutions des affaires des Chinois d'outre-mer et des organisations «politiques » de la diaspora, considérés à la fois comme facteurs et conséquences de la consolidation de la politique diasporique de la Chine (chapitre 7).

Un des apports de cette thèse réside dans le fait d'avoir montré qu'il n'existe pas qu'une organisation économique issue de la région de Wenzhou à proprement parler (chapitre 3) et qu'une organisation économique particulière prend forme au sein de la diaspora Wenzhou en France (chapitre 4). Dans ce contexte, nous avons pu aborder la métamorphose du "Modèle de Wenzhou " (chapitre 5). Cette organisation s'articule autour de la forte concentration géographique des entreprises tenues par les Wenzhou dans la région parisienne. L'explication d'une telle concentration de ces entreprises réside dans la mobilisation de leurs propres réseaux migratoires transnationaux, et dans le développement économique de leur pays d'origine. Ces réseaux sont également familiaux et reposent sur la concentration d'entreprises dans quelques secteurs d'activités commerciales ou productives. Ces secteurs économiques ont pu ensuite être qualifiés de secteurs transnationaux. La forme de l'organisation économique se réfère quant à elle principalement à des petites et moyennes entreprises (commerciales, de services ou de fabrication) qui ont joué et jouent encore un rôle économique non seulement à l'échelle locale (pratique du financement de type informel), mais aussi à l'échelle internationale (distribution des produits). En s'appuyant sur sa propre organisation économique et grâce à la mobilisation des réseaux transnationaux des migrants dans la distribution des produits, la diaspora Wenzhou permet la reproduction identitaire de son groupe comme corps social.

Cette recherche aborde un des groupes constitutifs de la diaspora chinoise comme une diaspora en elle-même. Ceci permet de contribuer à une réflexion plus générale sur l'articulation des diasporas (sous-groupes diasporiques, comme ici Wenzhou, Chaozhou, Hakka, etc.) au sein de la diaspora qui les englobe. La thèse enrichit la réflexion sur la notion de "diaspora entrepreneuriale " conçue comme collectif soit constitué de patrons ou de salariés. Elle se caractérise par l'existence d'un "marché ethnique du travail» rarement analysé, la thèse fournit de précieuses informations sur son fonctionnement.

16 Un des résultats est de montrer l'articulation forte entre réseaux migratoires et réseaux commerçants, au point qu'ils se superposent et se confondent. Le cas des migrants Wenzhou est emblématique dans la mesure où le modèle de Wenzhou repose, entre autres, sur la distribution de la production fabriquée à Wenzhou par la diaspora Wenzhou dans le reste de la Chine et dans le monde. Les activités économiques de la diaspora Wenzhou sont liées à une continuité dans l'internationalisation du développement économique de la région de Wenzhou, mais qu'elles sont aussi en lien étroit avec les différents pôles d'installation où se localisent leurs propres réseaux transnationaux.

17 Le transnationalisme est un autre thème auquel la thèse apporte sa contribution. Y sont analysés de manière approfondie les transferts de marchandises et financiers, mais aussi, et c'est tout à fait novateur, les transferts d'idées, de savoir-faire, d'innovations... bref de chose proprement immatérielles qui relèvent de ce que Peggy Levitt (2001) appelle les social remittances. Le point original dans la thèse porte sur la circulation de 
ce qu'on pourrait appeler des "modèles économiques» et donc de tout un ensemble spécifique de codes, normes et valeurs mobilisés dans les échanges marchands.

Les résultats de cette thèse permettent principalement de révéler l'existence d'une économie transnationale originale, reliant la France et la Chine, qui a été soutenue par la diaspora Wenzhou et a produit une forme de "transfert migratoire " dans chacun des deux pays. Comme nous l'avons vu plus haut une des questions était, s'il existe un "Modèle de Wenzhou » en Chine, retrouve-t-on ce même modèle en France ? Cette question nous a même servi de fil conducteur. Autrement dit, on s'interrogeait sur la possibilité d'une importation de ce modèle en France. À la fin de cette recherche nous en venons à nous demander au contraire s'il n'y a pas eu «transfert » vers la Chine d'une forme d'organisation économique créée en France par la diaspora Wenzhou qui s'y est établie.

Par ailleurs, il est important d'insister sur l'analyse politique livrée dans ce travail. Cette thèse montre avec beaucoup de finesse l'évolution des relations que peut entretenir la Chine avec sa diaspora. L'étude du changement de positionnement politique opéré par la Chine est un résultat important, qui nous donne à voir que la ligne stratégique du gouvernement à destination de la diaspora est passé d'un « revenir et servir le pays» à "servir le pays depuis l'étranger» (Ma Mung, 2008). Plus largement, la thèse permet de montrer de quelle manière la diaspora chinoise en France a contribué au développement économique de la Chine et comment elle a pu bénéficier de la nouvelle politique de la Chine initiée au début des années 2000 pour se consolider.

Enfin, cette thèse combine plusieurs approches et c'est ce qui en fait l'originalité : géographie du commerce et de l'entrepreneuriat ethnique, géographie des migrants, des diasporas et du transnationalisme, géographie urbaine et économique. Grâce à l'ensemble des données (données de première main sur terrain difficile d'accès, synthèse de données statistiques, une trentaine de cartes reposant sur de minutieux relevés de terrains) et avec une double approche par l'insertion dans la société d'installation et par les liens transnationaux entre la diaspora chinoise et la Chine, cette thèse montre comment des liens diasporiques et des circulations planétaires d'objets transforment les espaces urbains.

\section{BIBLIOGRAPHIE}

Auguin, Estelle (2009) L'éthique chinoise et l'esprit du capitalisme : la diaspora chinoise originaire de la région de Wenzhou, Paris, Université Paris Descarte, 423 p., Th. Doc : Sciolo. : Paris : 2009.

Chuang, Ya-Han (2015) Migrants chinois à Paris Au-delà de l'“intégration" : la formation politique d'une minorité, Paris, Université de Paris IV, 460 p.

Th. Doc. : Sociol. : Paris : 2015 
Freedman, Maurice (1957) Chinese Family and Marriage in Singapore, New York, Johnson Reprint Corp, 268 p.

Kuhn, Philip A. (2006) Why China Historians Should Study the Chinese Diaspora, and Vice-versa, Journal of Chinese Overseas , n², pp. 163-172.

Le Calloch, Bernard (1977) La diaspora chinoise en France, Acta Geographica, $n^{\circ} 30$.

Li, Minghuan. (2002) Ouzhouhuaqiaohuarenshi 欧洲华侨华人史 [A history of Chinese Immigrants in Europe], Beijing, Zhongguohuaqiaochubanshe 中国华侨出版社[The Chinese Overseas Publishing House], 860 p.

Liu, Hong (2009) Haiwaihuarenyanjiudepuxi : zhuti debianhua yu fangfadeyanjin 海外华人研究 的谱系：主题的变化与方法的演进 [Toward a Geneqlogy of Diasporic Chinese Studies : Changing Yhems and Evoling Approaches]. Huarenyanjiu guoji xuebao 华人研究国际学报 [Journal d'études des Chinois d'outre-mer], $\mathrm{n}^{\circ}$ 2, pp. 1-27.

Ma Mung, Emmanuel (2000) La diaspora chinoise : géographie d'une migration, Paris, OPHRYS, $175 \mathrm{p}$.

Ma Mung, Emmanuel (2008) Chinese Migration and China's Foreign Policy in Africa, Journal of Chinese overseas, $\mathrm{n}^{\circ} 1$, pp. 91-109.

Ma Mung, Emmanuel (2014) La diaspora chinoise en France, in Poinsot, M. ; Weber, S. (eds) Migration et mutations de la société française, Paris, La Découverte, pp. 121-129.

Peggy, Levitt (2001) The Transnational Villagers, Berkeley ; Los Angeles, Universty of California, $281 \mathrm{p}$.

Pina-Guerassimoff, Carine (2012) La Chine et sa nouvelle diaspora. La mobilité au service de la puissance, Paris, Ellipses, $237 \mathrm{p}$.

Poisson, Véronique (2004) Franchir les frontières : Le cas des Chinois du Zhejiang en diaspora, Paris, Ecole des Hauts Etudes en Sciences sociales, 903 p., Th. Doc. : Anthro : Paris : 2004

Polyzos, Iris (2014) Parcours des migrants et mutations sociospatiales à Athènes : le cas des commerçants chinois à Metaxourgio, Athènes ; Poitiers, Université Technique nationale d'Athènes ; Université de Poitiers, 335 p.

Th. Doc. : Géogr, Athènes :2014.

Purcell, Victor (1965) The Chinese in Southeast Asia, Oxford, Oxford University Press, $654 \mathrm{p}$.

Shi, Jinchuan; Kangdui, Zhu (2001) Wenzhoumoshi yanjiu : huigu yuzhanwang 温州模式研究 : 回顾与展望 [Recherches sur le 《 Modèle de Wenzhou » : Histoire et perspective], Wenzhouluntan 温州论坛 [Forum de Wenzhou], n5, pp. 10-20.

Skinner, George William (1957) Chinese Society in Thailand : An Analytical History, London, Cornell University Press, $490 \mathrm{p}$.

Thunø, Mette ; Pieke, Frank N. (2005) Institutionalizing Recent Rural Emigration from China to Europe : New Transnational Villages in Fujian, International Migration Review, $n^{\circ} 2$, pp. 485-514.

Trolliet, Pierre (1994) La diaspora chinoise, Paris, Presses universitaires de France, 126 p. (Que sais-je ?).

Wang, Gungwu (1991) China and the Chinese Overseas, Times Academic Press, 328 p.

Wang, Gungwu (2000) A Single Chinese Diaspora?, Joining the Modern World : Inside and Outside China, World Scientific, pp. 37-70. 
Wang, Gungwu (2006) Patterns of Chinese Immigration in Historical Perspective, in Hong Liu (ed.) The Chinese Overseas vol. 1, London, Taylor \& Francis, 456 p.

Wang, Simeng (2014) Expériences migratoires au prisme des usages des soins psychiatriques. Le cas de l'immigration chinoise en région parisienne : Une enquête ethnographique en institution et dans les familles, Paris, École des Hauts Études en Sciences sociales, 464 p.

Th. Doc. : Socio : Paris : 2014

Wickberg, Edgar (1965) The Chinese in Philippine Life, 1850-1898, Manila, Ateneo University Press, $302 \mathrm{p}$.

Zhou, Min (1992) Chinatown : The Socioeconomic Potential of an Urban Enclave, Philadelphia, Temple University Press, 275 p.

Zhuang, Guotu (2009) Dongnanya huaqiaohuaren shuliang de xingusuan 东南亚华侨华人数量的 新估算[La nouvelle évaluation du nombre des Chinois d'outre-mer en Asie du Sud-Est], Xiamen daxue xuebao (zexueshehuikexueban) 厦门大学学报（哲学社会科学版) [Journal of Xiamen University (Arts\&Social Sciences)], $n^{\circ}$ 193, pp. 62-69.

\section{NOTES}

1. Signalons toutefois deux thèses récemment soutenues celle de Wang Simeng en juillet 2014 et celle Chuang Ya-han en novembre 2015.

2. C'est une théorie née au XXe siècle qui explique le phénomène du mouvement de la migration concernant un effet de pression (push) dans le lieu de départ et un effet d'attraction (pull) dans le lieu d'arrivé. Aujourd'hui, cette théorie a été critiquée beaucoup par plusieurs chercheurs, par exemple elle négocie le fait de la subjectivité pour un migrant (Li, 2002).

\section{INDEX}

Index géographique : Chine, France

Mots-clés : diaspora, commerce, entreprenariat ethnique, transnationalisme, recherche, étude de cas, Wenzhou

\section{AUTEURS}

\section{ZHIPENG LI}

Docteur en géographie, MIGRINTER-Université de Poitiers

Chercheur post-doctorant, Projet Émergence(s) - CERI

zp.li@sciencespo.fr 